TRANSACTIONS OF THE

AMERICAN MATHEMATICAL SOCIETY

Volume 361, Number 10, October 2009, Pages 5457-5468

S 0002-9947(09)04721-7

Article electronically published on April 21, 2009

\title{
THE TOPOLOGY OF SYMPLECTIC CIRCLE BUNDLES
}

\author{
JONATHAN BOWDEN
}

\begin{abstract}
We consider circle bundles over compact three-manifolds with symplectic total spaces. We show that the base of such a space must be irreducible or the product of the two-sphere with the circle. We then deduce that such a bundle admits a symplectic form if and only if it admits one that is invariant under the circle action in three special cases: namely, if the base is Seifert fibered, has vanishing Thurston norm, or if the total space admits a Lefschetz fibration.
\end{abstract}

\section{INTRODUCTION}

A conjecture due to Taubes states that if a closed, compact 4-manifold of the form $M \times S^{1}$ is symplectic, then $M$ must fiber over $S^{1}$. A natural extension of this conjecture is to the case where $E \stackrel{\pi}{\rightarrow} M$ is a possibly nontrivial circle bundle. In [4] it was shown that an $S^{1}$-bundle admits an $S^{1}$-invariant symplectic form if and only if its base fibers over $S^{1}$ and the Euler class $e(E)$ of the total space pairs trivially with the fiber of some fibration. Thus based on the principle that an $S^{1}$-bundle should admit a symplectic form if and only if it admits an invariant one, one arrives at the following conjecture.

Conjecture 1 (Taubes). If a circle bundle $S^{1} \rightarrow E \stackrel{\pi}{\rightarrow} M$ over a closed, oriented 3-manifold is symplectic, then there is a fibration $\Sigma \rightarrow M \stackrel{\phi}{\rightarrow} S^{1}$ such that $e(E)([\Sigma])=0$.

If an oriented 3-manifold fibers over $S^{1}$ with fiber $\Sigma \neq S^{2}$, then it follows by the long exact homotopy sequence that $M$ is in fact aspherical. So a necessary condition for Conjecture 1 to hold is that any $M$ that is the base of an $S^{1}$-bundle, whose total space carries a symplectic form, must in fact be aspherical or $S^{2} \times S^{1}$ in the case $\Sigma=S^{2}$. This observation provides the motivation for the following theorem, which is the main result of the first part of this paper.

Theorem 2, Let $M$ be an oriented, closed 3-manifold so that some circle bundle $S^{1} \rightarrow E \stackrel{\pi}{\rightarrow} M$ admits a symplectic structure. Then, either $M$ is diffeomorphic to $S^{2} \times S^{1}$ and the bundle is trivial, or $M$ is irreducible and aspherical.

A similar statement was proved by McCarthy in [20] for the case $E=M \times S^{1}$. More precisely, McCarthy showed that if $M \times S^{1}$ admits a symplectic structure,

Received by the editors November 19, 2007.

2000 Mathematics Subject Classification. Primary 57R17; Secondary 57N10, 57N13.

(C)2009 American Mathematical Society Reverts to public domain 28 years from publication 
then $M$ decomposes as a connected sum $M=A \# B$, where the first Betti number $b_{1}(A) \geq 1$ and $B$ has no nontrivial connected covering spaces. This can be refined quite substantially following Perelman's proof of Thurston's geometrisation conjecture (see [22, 23] or [21]), for one corollary of geometrisation is that the fundamental group of a closed 3-manifold is residually finite (see [1]), meaning that the $B$ in McCarthy's theorem must have trivial fundamental group, and hence by the Poincaré Conjecture is diffeomorphic to $S^{3}$. Thus in fact $M$ must be prime and hence irreducible and aspherical or $S^{2} \times S^{1}$. Theorem 2 is then a generalisation of this more refined statement to the case of nontrivial $S^{1}$-bundles. Our argument will rely on a vanishing result of Kronheimer-Mrowka for the Seiberg-Witten invariants of a manifold that splits into two pieces along a copy of $S^{2} \times S^{1}$, which in itself is of independent interest (cf. Proposition 1). One may also prove Theorem 2 by following the argument of [20]; see Remark 1 below.

In the remainder of this paper we will show that Conjecture 1 holds in various special cases. First we will verify the conjecture under certain additional assumptions on the topology of the base manifold $M$. In order to be able to do this we will need to understand when a manifold fibers over $S^{1}$. One gains significant insight into this problem by considering the Thurston norm \|\|$_{T}$ on $H^{1}(M, \mathbb{R})$, which was introduced by Thurston in [27. The Thurston norm enables one to see which integral classes $\alpha \in H^{1}(M, \mathbb{Z})$ can be represented by closed, nonvanishing 1-forms, which in turn induce fibrations of $M$ by compact surfaces.

In [5] Friedl and Vidussi showed that if $E=M \times S^{1}$ admits a symplectic form and \|\|$_{T} \equiv 0$ or $M$ is Seifert fibered, then $M$ must fiber over $S^{1}$. In Corollary 2 below we will show that in fact Conjecture 1 holds in these two cases. The argument will be based on understanding the Seiberg-Witten invariants of the total space $E$ given that $M$ has vanishing Thurston norm and the Seifert case will be deduced as a corollary of this. Indeed, if $M$ has vanishing Thurston norm and $S^{1} \rightarrow E \stackrel{\pi}{\rightarrow} M$ is symplectic, then the canonical class of $E$ must be trivial. This combined with the restrictions on Seiberg-Witten basic classes of a symplectic manifold as proved by Taubes in [26] means that $K=0$ is the only Seiberg-Witten basic class and the result then follows by an application of a vanishing result of Lescop (cf. 17] or [28]).

Another special case of the Taubes conjecture is when the total space $E$ admits a Lefschetz fibration, as was considered in 2 and 3 for a trivial bundle. In view of Corollary 2 we will be able to give a comparatively simple proof of the following result.

Theorem [9. Let $S^{1} \rightarrow E \stackrel{\pi}{\rightarrow} M$ be a symplectic circle bundle over an irreducible base $M$. If $E$ admits a Lefschetz fibration, then $M$ fibers over $S^{1}$.

It then follows by considering the Kodaira classification of complex surfaces that Conjecture 1 holds under the assumption that the total space admits a complex structure.

Outline of paper. In Section 2 we will state the relevant vanishing result of Kronheimer-Mrowka in order to prove Theorem 2. In Section 3 we recall the definition of the Thurston norm and quote some well-known facts about it. In Section 4 we will use our knowledge of the Thurston norm to verify Conjecture 1 under the assumption that the base is Seifert fibered or has vanishing Thurston norm. Finally in Section [5] we will define Lefschetz fibrations and prove that $M$ is fibered when one has a Lefschetz fibration on the total space $E$. 


\section{Asphericity OF The BASE $M$}

Throughout this article all manifolds will be closed, connected and oriented and $M$ will always denote a manifold of dimension 3 . In addition we will make the convention that all (co)homology groups will be taken with integral coefficients unless otherwise stated.

In 20] it was shown that if $M \times S^{1}$ is symplectic, then $M$ must be irreducible and aspherical or $S^{2} \times S^{1}$. We extend this to the case of a nontrivial $S^{1}$-bundle. We first collect some relevant lemmas.

Lemma 1. Let $M=M_{1} \# M_{2}$ be a nontrivial connect sum decomposition with $b_{1}(M) \geq 1$. Then there is a finite covering $N$ of $M$ that decomposes as a direct sum $N=N_{1} \# N_{2}$ where $b_{1}\left(N_{i}\right) \geq k$ for any given $k$.

Proof. It follows from Mayer-Vietoris that the Betti numbers are additive for a connect sum; hence by assumption we may assume that $b_{1}\left(M_{1}\right) \geq 1$. By the proof of geometrisation it follows that the fundamental group of a 3-manifold is residually finite (cf. [11]) and hence $M_{2}$ has a nontrivial $d$-fold cover $\tilde{M}_{2}$, with $d \geq 2$. By removing a ball from $M_{2}$ and its disjoint lifts from $\tilde{M}_{2}$ and then gluing in $d$ copies of $M_{1}$ we obtain a cover $\tilde{M}$ of $M=M_{1} \# M_{2}$, and by construction $\tilde{M}$ has a connect sum decomposition as $\tilde{M}=M_{1} \# P$, where $b_{1}(P) \geq 1$. We may now take a $k$ fold cover associated to some surjective homomorphism of $\pi_{1}\left(M_{1}\right) \rightarrow \mathbb{Z}_{k}$ and glue in copies of $P$ to get a cover of $\tilde{M}$ (and hence of $M$ ), which decomposes in two pieces one of which has first Betti number at least $k$. One more application of this procedure gives the desired result.

Lemma 2. Let $S^{1} \rightarrow E \stackrel{\pi}{\rightarrow} M$ be a circle bundle, whose Euler class we denote by $e(E) \in H^{2}(M)$. Then

(1) $b_{2}(E)= \begin{cases}2 b_{1}(M)-2 & \text { if } e(E) \text { is not torsion, } \\ 2 b_{1}(M) & \text { if } e(E) \text { is torsion. }\end{cases}$

(2) $b_{2}^{+}(E)=b_{2}^{-}(E) \geq b_{1}(M)-1$.

Proof. We consider the Gysin sequence

$$
H^{0}(M) \stackrel{\cup e}{\rightarrow} H^{2}(M) \stackrel{\pi^{*}}{\rightarrow} H^{2}(E) \stackrel{\pi_{*}}{\rightarrow} H^{1}(M) \stackrel{\cup e}{\rightarrow} H^{3}(M),
$$

where $e \in H^{2}(M)$ denotes the Euler class of the bundle. By Poincaré duality $H^{0}(M)=H^{3}(M)=\mathbb{Z}$ and $b_{1}(M)=b_{2}(M)$, so we conclude by exactness that $b_{2}(E)=2 b_{1}(M)-2$ if $e$ is not torsion and $b_{2}(E)=2 b_{1}(M)$ if $e$ is torsion. Furthermore since $E$ bounds its associated disc bundle, it has zero signature and hence

$$
b_{2}^{+}(E)=b_{2}^{-}(E) \geq b_{1}(M)-1
$$

We will need to appeal to a vanishing result for the Seiberg-Witten invariants of manifolds that decompose along $S^{2} \times S^{1}$, which we take from [16]. For this we will need to define a relative notion of $b_{2}^{+}$for an oriented 4 -manifold $X$ with boundary. This is done by considering the symmetric form induced on rational cohomology that is obtained as the composition

$$
H^{2}(X, \partial X) \times H^{2}(X, \partial X) \stackrel{i^{*} \times I d}{\rightarrow} H^{2}(X) \times H^{2}(X, \partial X) \stackrel{\cup}{\rightarrow} \mathbb{Q} .
$$

Here the map $i^{*}$ is the map coming from the long exact sequence of the pair $(X, \partial X)$ and the second map is nondegenerate by Poincaré duality. This is then a symmetric, 
possibly degenerate, form on $H^{2}(X, \partial X)$ and we define $b_{2}^{+}(X)$ to be the dimension of a maximal positive definite subspace.

Theorem 1 (Kronheimer-Mrowka, [16]). Let $X=X_{1} \cup_{\partial X_{1}=\partial X_{2}} X_{2}$ where $\partial X_{1}=$ $-\partial X_{2}=S^{2} \times S^{1}$ and $b_{2}^{+}\left(X_{1}\right), b_{2}^{+}\left(X_{2}\right) \geq 1$. Then for all Spin ${ }^{c}$-structures $\xi$,

$$
\sum_{\xi^{*}-\xi \in \text { Tor }} \mathrm{SW}\left(\xi^{*}\right)=0 .
$$

Although it is not explicitly stated in the book [16, Theorem 1 can be deduced as follows: formula 3.27 (p. 75) allows one to compute the sum of the SW invariants of all $S_{p i n}{ }^{c}$-structures that differ by torsion as a pairing of certain Floer groups. However these groups are zero for $S^{2} \times S^{1}$ by Proposition 3.10.3 in the case of an untwisted coefficient system and by Proposition 3.10.4 in the twisted case, and thus this sum must vanish.

Theorem 1 then implies certain restrictions on the decomposition of symplectic manifolds along a copy of $S^{2} \times S^{1}$.

Proposition 1. A symplectic manifold $X$ cannot be decomposed as $X=$ $X_{1} \cup_{\partial X_{1}=\partial X_{2}} X_{2}$, where $\partial X_{1}=-\partial X_{2}=S^{2} \times S^{1}$ and $b_{2}^{+}\left(X_{1}\right), b_{2}^{+}\left(X_{2}\right) \geq 1$.

Proof. By the hypotheses of the proposition, we conclude from Theorem 1 that for every $\operatorname{Spin}^{c}$-structure $\xi \in \operatorname{Spin}^{c}(X)$,

$$
\sum_{\xi^{*}-\xi \in T o r} \operatorname{SW}\left(\xi^{*}\right)=0
$$

However as $X$ is symplectic and

$$
b_{2}^{+}(X) \geq b_{2}^{+}\left(X_{1}\right)+b_{2}^{+}\left(X_{2}\right) \geq 2
$$

the nonvanishing result of Taubes implies $\mathrm{SW}\left(\xi_{\text {can }}\right)= \pm 1$, where $\xi_{\text {can }}$ denotes the canonical Spinc ${ }^{c}$-structure associated to the symplectic structure on $E$ (cf. [25]). Moreover it follows from the constraints on SW basic classes of a symplectic manifold of [26] that if $\xi^{*}$ is another Spin ${ }^{c}$-structure with nontrivial SW invariant and $\xi_{c a n}-\xi^{*} \in T o r$, then in fact $\xi_{c a n}=\xi^{*}$. Hence

$$
\sum_{\xi^{*}-\xi_{c a n} \in T o r} \operatorname{SW}\left(\xi^{*}\right)= \pm 1
$$

which is a contradiction.

Theorem 2. Let $M$ be an oriented, closed 3-manifold, so that some circle bundle $S^{1} \rightarrow E \stackrel{\pi}{\rightarrow} M$ admits a symplectic structure. Then $M$ is irreducible and aspherical or $M=S^{2} \times S^{1}$ and the bundle is trivial.

Proof. We first show that $M$ must be prime. Since $E$ is symplectic it follows from Lemma 2 that $b_{1}(M) \geq 1$. Assume that $M=M_{1} \# M_{2}$ is a nontrivial connected sum. Then by taking a suitable covering as in Lemma 1 and pulling back $E$ and its symplectic form we may assume without loss of generality that $b_{1}\left(M_{i}\right) \geq 2$. We let $S$ denote the gluing sphere of the connected sum. Then as $S$ is nullhomologous the bundle restricted to $S$ is trivial. Thus the connect sum decomposition induces a decomposition $E=E_{1} \cup_{S^{2} \times S^{1}} E_{2}$. Since the bundles $E_{i} \rightarrow M_{i} \backslash B^{3}$ are trivial on the boundary we may extend them to bundles $\tilde{E}_{i} \rightarrow M_{i}$ and as $b_{1}\left(M_{i}\right) \geq 2$, Lemma 2 implies that $b_{2}^{+}\left(\tilde{E}_{i}\right) \geq 1$. Further, since $E_{i} \simeq \tilde{E}_{i} \backslash\left(S^{1} \times p t\right)$ we have that

$$
b_{2}^{+}\left(E_{i}\right) \geq b_{2}^{+}\left(\tilde{E}_{i}\right) \geq 1
$$


which then contradicts Proposition 1. Hence $M$ is prime, and thus irreducible or $S^{2} \times S^{1}$.

We assume that $M$ is irreducible. Then by the sphere theorem, $\pi_{2}(M)=0$. Since $b_{1}(M) \geq 1$, we have that $\pi_{1}(M)$ is infinite, so the universal cover $\tilde{M}$ of $M$ is not compact and has $\pi_{i}(\tilde{M})$ trivial for $i=1,2$. The Hurewicz theorem then implies that the first nontrivial $\pi_{i}(\tilde{M})$ is isomorphic to $H_{i}(\tilde{M})$. But since $\tilde{M}$ is not compact, $H_{3}(\tilde{M})=0$ and as $\tilde{M}$ is 3-dimensional, $H_{i}(\tilde{M})=0$ for all $i \geq 4$. Hence $\pi_{i}(\tilde{M})=0$ for all $i \geq 1$ and it follows from Whitehead's Theorem that $\tilde{\tilde{M}}$ is contractible, that is, $M$ is aspherical.

In the case where $M=S^{2} \times S^{1}$, any symplectic bundle must be trivial by Lemma 2 .

Remark 1 . One may also give a proof of Theorem2 that uses the covering construction of [20. In order to do this one first takes finite coverings on each of the two pieces in the connect sum decomposition. Then one glues these together to find a covering $\tilde{M}$ where the sphere of the connect sum lifts to a sphere that is nontrivial in real cohomology. This sphere then lifts to the total space of the pullback bundle $\tilde{E}$ over $\tilde{M}$. One may also assume by Lemma 1 that $b_{1}(\tilde{M})$ is large and hence $b_{2}^{+}(\tilde{E})$ is large. Then a standard vanishing theorem for the SW invariants (cf. 13]) implies that all invariants are zero, which then contradicts Taubes' result if $E$ and hence $\tilde{E}$ is symplectic.

By considering the long exact homotopy sequence we have the following corollary that was first proved by Kotschick in [14].

Corollary 1. Let $S^{1} \rightarrow E \stackrel{\pi}{\rightarrow} M$ be a symplectic circle bundle over an oriented 3-manifold $M$. Then the map $\pi_{1}\left(S^{1}\right) \rightarrow \pi_{1}(E)$ induced by the inclusion of the fiber is injective. In particular a fixed point free circle action on a symplectic 4-manifold can never have contractible orbits.

\section{The Thurston NORM}

In this section we will define and collect several relevant facts about the Thurston norm. We first define the negative Euler characteristic or complexity of a possibly disconnected, orientable surface $\Sigma=\bigsqcup_{i} \Sigma_{i}$ to be

$$
\chi_{-}(\Sigma)=\sum_{\chi\left(\Sigma_{i}\right) \leq 0}-\chi\left(\Sigma_{i}\right)
$$

where $\chi$ denotes the Euler characteristic of the surface.

Next we define the Thurston norm \|\|$_{T}$ as a map on $H^{1}(M)$ by

$$
\|\sigma\|_{T}=\min \left\{\chi_{-}(\Sigma) \mid P D(\Sigma)=\sigma\right\} .
$$

It is a basic fact that this map extends uniquely to a (semi)norm on $H^{1}(M, \mathbb{R})$, which we will denote again by \|\|$_{T}$. One particularly important property of the Thurston norm is that its unit ball, which we denote by $B_{T}$, is a (possibly noncompact) convex polytope with finitely many faces. If $B_{T^{*}}$ denotes the unit ball in the dual space we have the following characterisation of $B_{T}$.

Theorem 3 (27], p. 106). The unit ball $B_{T^{*}}$ is a polyhedron whose vertices are integral lattice points, $\pm \beta_{1}, \ldots, \pm \beta_{k}$ and the unit ball $B_{T}$ is defined by the following inequalities:

$$
B_{T}=\left\{\alpha|| \beta_{i}(\alpha) \mid \leq 1,1 \leq i \leq k\right\} .
$$


We are interested in understanding how a manifold fibers over $S^{1}$, and the following theorem says that the Thurston norm determines precisely which cohomology classes can be represented by fibrations.

Theorem 4 ([27], p. 120). Let $M$ be a compact, oriented 3-manifold. The set $F$ of cohomology classes in $H^{1}(M, \mathbb{R})$ representable by nonsingular closed 1 -forms is the union of the open cones on certain top-dimensional open faces of $B_{T}$, minus the origin. The set of elements in $H^{1}(M, \mathbb{Z})$ whose Poincaré dual is represented by the fiber of some fibration consists of the set of lattice points in $F$.

We call a top-dimensional face of the unit ball $B_{T}$ fibered if some integral class, and hence all, in the cone over its interior can be represented by a fibration. One also understands how the Thurston norm behaves under finite covers by the following result of Gabai.

Theorem 5 ([7], Cor. 6.13). Let $\tilde{M} \stackrel{p}{\rightarrow} M$ be a finite connected $d$-sheeted covering. Then for $\sigma \in H^{1}(M, \mathbb{R})$ we have

$$
\|\sigma\|_{T}=\frac{1}{d}\left\|p^{*} \sigma\right\|_{T}
$$

These facts then allow us to completely characterise the Thurston norm of an irreducible Seifert fibered manifold.

Proposition 2. If $M$ is irreducible and Seifert fibered, then either the Thurston norm of $M$ vanishes identically or $M$ fibers over $S^{1}$ and

$$
\|\sigma\|_{T}=m \cdot|\sigma(\gamma)|
$$

for some class $\gamma \in H_{1}(M)$.

Proof. Since $M$ is irreducible and Seifert fibered, either $M$ has a horizontal surface, i.e. a closed surface transverse to all fibers, or every embedded surface is isotopic to a vertical surface, i.e. a surface that is a union of fibers (cf. [9], Prop 1.11) and is hence a union of tori, so the Thurston norm is identically zero. If $M$ has a horizontal surface $F$, which we may assume to be connected, then $M$ is a mapping torus with monodromy $\phi \in \operatorname{Diff}^{+}(F)$ so that $\phi^{n}=I d$ for some $n$. This means that $M$ is covered by $\tilde{M}=F \times S^{1}$. If $\tilde{\gamma}=p t \times S^{1}$, then the Thurston norm of $\tilde{M} \stackrel{p}{\rightarrow} M$ is given by

$$
\|\sigma\|_{T}=\chi_{-}(F)|\sigma(\tilde{\gamma})|
$$

We let $\gamma=p_{*}(\tilde{\gamma})$. Then by Theorem 5 the norm on $M$ is given by

$$
\|\sigma\|_{T}=\frac{1}{n}\left\|p^{*} \sigma\right\|_{T}=\frac{\chi_{-}(F)}{n}\left|p^{*} \sigma(\tilde{\gamma})\right|=\frac{\chi_{-}(F)}{n}|\sigma(\gamma)|=m .|\sigma(\gamma)| .
$$

Example 1 (Seifert fibered spaces with horizontal surfaces). We note that in the second case of Proposition 2 the Thurston ball $B_{T}$ consists of two (noncompact) faces that are both fibered and that the Thurston norm is identically zero on a codimension one subspace $K$. Thus by [4] any bundle over such an $M$ will admit an $S^{1}$-invariant symplectic form except possibly in the case where the Euler class $e(E)$ pairs trivially with all elements in $K$; that is, $e(E)$ pairs trivially with all tori in $M$. By taking the pullback bundle of the cover $\tilde{M}=F \times S^{1} \rightarrow M$ we may assume that we have a bundle $E$ over $F \times S^{1}$ that is symplectic and has Euler class that again pairs trivially with embedded tori in $\tilde{M}$ and is thus a nonzero multiple of 
$P D(\tilde{\gamma})$. This in turn has a covering $\bar{E}$ that is an $S^{1}$-bundle with Euler class equal to $P D(\tilde{\gamma})$. Now if we let $T=\tilde{\gamma} \times S^{1}$ and $X=\tilde{M} \times S^{1}$, then the SW polynomial of $X$ can be computed to be

$$
\mathcal{S W}_{X}^{4}=\left(t_{T}-t_{T}^{-1}\right)^{2 g-2},
$$

where $g$ is the genus of $F$. Then by the formula of Baldridge in [1, it follows that all the SW invariants of $\bar{E}$ are zero, contradicting Taubes' nonvanishing result for the SW invariants of a symplectic manifold. So in fact Conjecture 1 holds for Seifert fibered spaces that have horizontal surfaces.

\section{The Case of Vanishing Thurston norm}

In [5] Friedl and Vidussi showed that if $E=M \times S^{1}$ admits a symplectic form and \|\|$_{T} \equiv 0$ or $M$ is Seifert fibered, then $M$ must fiber over $S^{1}$. In this section we shall extend this to the case of a nontrivial $S^{1}$-bundle and then show that Conjecture 1 holds in both of these cases. From now on we shall assume that $M$ is irreducible, which in view of Theorem 2 only excludes the case where $M=S^{2} \times S^{1}$ and the bundle is trivial. Our argument will be based on that of [5], and we begin with the following lemma.

Lemma 3. If $S^{1} \rightarrow E \stackrel{\pi}{\rightarrow} M$ is a bundle over an $M$ that has vanishing Thurston norm, then

$$
H^{2}(E) / T o r=V \oplus W,
$$

where $V, W$ are isotropic subspaces that admit a basis of embedded tori.

Proof. We consider the Gysin sequence

$$
\mathbb{Z} \longrightarrow H^{2}(M) \stackrel{\pi^{*}}{\longrightarrow} H^{2}(E) \underset{s}{\stackrel{\pi_{*}}{\rightleftarrows}} H^{1}(M) \longrightarrow \mathbb{Z} .
$$

Here $s$ is a section defined on the image of $\pi_{*}$ as follows: we represent an element $\sigma \in H^{1}(M)$ by an embedded surface $\Sigma$. By exactness, $\sigma$ will be in $\operatorname{Im}\left(\pi_{*}\right)$ precisely when the bundle is trivial on $\Sigma$ and in this case we may lift $\Sigma$ to some $\tilde{\Sigma}$ in $E$. As $H^{1}(M)$ is free, we define $s$ on a $\mathbb{Z}$-basis $\left\{\sigma_{i}\right\}$ by $s\left(\sigma_{i}\right)=\tilde{\Sigma}_{i}$. We set $V=\pi^{*}\left(H^{2}(M)\right)$ and $W=s\left(H^{1}(M)\right)$. Then $V$ is clearly spanned by embedded tori and the statement for $W$ is precisely the assumption on the Thurston norm.

Proposition 3. Let $S^{1} \rightarrow E \stackrel{\pi}{\rightarrow} M$ be an $S^{1}$-bundle with torsion Euler class $e(E)$. Then there is a finite cover $\tilde{M} \stackrel{p}{\rightarrow} M$ such that the pullback bundle $p^{*} E \rightarrow \tilde{M}$ is trivial.

Proof. We choose a splitting of $H_{1}(M)=F \oplus T$, where $T$ is the torsion subgroup and $F$ is any free complement. We take the cover $\tilde{M} \stackrel{p}{\rightarrow} M$ associated to the kernel of the composition

$$
\pi_{1}(M) \rightarrow H_{1}(M) \stackrel{\phi}{\rightarrow} T
$$

where $\phi$ is the projection with kernel $F$. Note that the composition $H_{1}(\tilde{M}) \stackrel{p_{*}}{\rightarrow}$ $H_{1}(M) \stackrel{\phi_{*}}{\rightarrow} T$ is zero. Then by the Universal Coefficient Theorem we have the 
following commutative diagram:

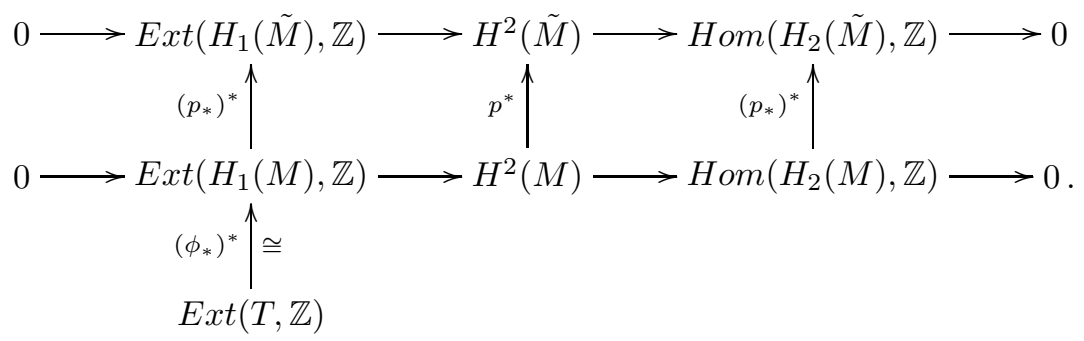

This implies that $p^{*}$ is zero on torsion in $H^{2}(M)$, so the pullback bundle is indeed trivial.

Theorem 6. If $S^{1} \rightarrow E \stackrel{\pi}{\rightarrow} M$ is a symplectic circle bundle over an irreducible manifold for which \|\|$_{T}$ is identically zero, then $M$ fibers over $S^{1}$.

After this paper had been submitted the author was informed that Friedl and Vidussi have independently proved this result (cf. [6]).

Proof. Since $E$ is symplectic it has an associated canonical $S p i n^{c}$-structure $\xi_{c a n}$ and canonical class that we denote by $K$. We claim that our assumption on the Thurston norm of the base implies that $K$ must be torsion. For by Taubes' nonvanishing result $\xi_{\text {can }}$ has nontrivial SW invariant. If $\alpha \in H^{2}(E)$, the adjunction inequality (see [15]) and Lemma 3 imply that

$$
|\alpha . K|=0 .
$$

This also holds in the case $b_{2}^{+}(E)=1$ (cf. [18, Theorem E and [19, Theorem B). As $M$ is irreducible and $b_{2}(M) \geq 1$ the assumption on the vanishing of the Thurston norm implies that $M$ contains an embedded, incompressible torus $T \hookrightarrow M$. Then by Proposition 7 of [12] either $T$ is the fiber of some fibration or there is a finite cover $\bar{M} \stackrel{p}{\rightarrow} M$ with large $b_{1}$, say $b_{1}(\bar{M}) \geq 4$. We assume that the latter holds. Then the pullback $\bar{E}=p^{*} E$ will be symplectic with canonical class $\bar{K}=p^{*} K$, with symplectic form $\bar{\omega}=p^{*} \omega$ and $b_{2}^{+}(\bar{E}) \geq 2$. Then for any $\operatorname{Spin}^{c}$-structure $\xi_{\text {can }} \otimes F$ that has nontrivial SW invariant we have by [26],

$$
0 \leq F \cdot[\bar{\omega}] \leq \bar{K} \cdot[\bar{\omega}] .
$$

Moreover, since $\bar{K}$ is torsion and equality on the left implies $F=0$, we conclude that in fact $\bar{K}=0$. Thus $\bar{K}=0$, so $\bar{\xi}_{\text {can }}$ is trivial and this is the only Spincstructure with nonzero SW invariant. We now need to consider two cases. We first assume that $e(E)$ and hence $e(\bar{E})$ is nontorsion. In this case we compute

$$
\pm 1=\sum_{\xi^{*} \in \operatorname{Spin}^{c}(\bar{E})} S W_{\bar{E}}^{4}\left(\xi^{*}\right)=\sum_{\xi^{*} \in \operatorname{Spin}^{c}(\bar{E})} \sum_{\xi^{*} \equiv \xi \bmod \bar{e}} S W_{\bar{M}}^{3}(\xi)=\sum_{\xi \in S p i n^{c}(\bar{E})} S W_{\bar{M}}^{3}(\xi),
$$

where the second inequality follows from Theorem 1 in [1. However as $b_{1}(\bar{M}) \geq 4$ this sum is zero (cf. [28, p. 114), a contradiction. If the Euler class is torsion we may assume by Proposition 3 that it is indeed zero and the above calculation reduces to

$$
\pm 1=\sum_{\xi \in \operatorname{Spin}^{c}(\bar{E})} S W_{\bar{E}}^{4}(\xi)=\sum_{\xi \in \operatorname{Spin}^{c}(\bar{E})} S W_{\bar{M}}^{3}(\xi)=0 .
$$

In either case we obtain a contradiction and hence $M$ must fiber over $S^{1}$. 
As a consequence of this theorem we conclude that Conjecture 1 holds if $M$ has vanishing Thurston norm or is Seifert fibered.

Corollary 2. Conjecture 1 holds if $M$ is Seifert fibered or \|\|$_{T} \equiv 0$.

Proof. If $M$ has vanishing Thurston norm, then by Theorem 4 we conclude that if one class in $H^{1}(M)$ can be represented by a fibration, then so can all classes and by the construction of [4 every bundle over $M$ admits an $S^{1}$-invariant symplectic form. If $M$ is Seifert fibered it either has vanishing Thurston norm by Proposition 2 and we proceed as in the previous case or $M$ has a horizontal surface and the claim follows by Example 1 above.

\section{The CASE Where $E$ ADMits a Lefschetz Fibration}

In 2. Chen and Matveyev showed that if $S^{1} \times M$ admits a symplectic Lefschetz fibration, then $M$ fibers over $S^{1}$. This was extended by Etgü in [3] to the case where the fibration may or may not be symplectic. In this section we shall show that the same statement holds for arbitrary $S^{1}$-bundles. Let us begin with some definitions and basic facts concerning Lefschetz fibrations.

Definition 7. Let $E$ be a compact, connected, oriented smooth 4-manifold. A Lefschetz fibration is a map $E \stackrel{p}{\rightarrow} B$ to an orientable surface so that any critical point has an oriented chart on which $p\left(z_{1}, z_{2}\right)=z_{1}^{2}+z_{2}^{2}$.

We list some basic properties of Lefschetz fibrations (for proofs see [8]).

(1) There are finitely many critical points, so the generic preimage of a point will be a surface and we may assume that this is connected. To each critical point one associates a vanishing cycle in the fiber.

(2) A Lefschetz fibration admits a symplectic form so that the fiber is a symplectic submanifold if the class $[F]$ of the fiber is nontorsion in $H_{2}(E)$. Moreover this is always true if $\chi(F) \neq 0$.

(3) We have a formula for the Euler characteristic given by

$$
\chi(E)=\chi(B) \cdot \chi(F)+\#\{\text { critical points }\} .
$$

We will first show that for a symplectic circle bundle any Lefschetz fibration will actually be a proper fibration, i.e. cannot have any critical points. The following lemma is essentially Lemma 3.4 of [2].

Lemma 4. Let $S^{1} \rightarrow E \stackrel{\pi}{\rightarrow} M$ be a circle bundle that admits a Lefschetz fibration $E \stackrel{p}{\rightarrow} B$. Then $p$ has no critical points.

Proof. We first consider the case where $F=S^{2}$. Since $E$ is spin, it has an even intersection form and thus all vanishing cycles are nonseparating in the fiber $F=$ $S^{2}$. However this means there cannot be any since $S^{2}$ is simply connected and hence $p$ has no critical points.

If $F=T^{2}$, the equation

$$
0=\chi(E)=\chi(B) \cdot \chi(F)+\#\{\text { critical points }\}
$$

implies that $E$ has no critical points.

We now consider the case when $F$ has genus greater than 1 . We know that $E$ admits a symplectic Lefschetz fibration by (2) above. Thus by the adjunction formula for symplectic surfaces we see that

$$
K . F=\chi_{-}(F) \neq 0,
$$


where $K$ is the canonical class on $E$. If $b_{2}^{+}>1$, then it follows from Taubes' result that $K$ is a basic class and thus the adjunction inequality holds. In the case where $b_{2}^{+}(E)=1$ we may apply the adjunction inequality exactly as in the case of $b_{2}^{+}>1$ by ([18], Theorem E). Now we assume that our fibration has a critical point and hence a vanishing cycle $\gamma$. Then we know that this is nonseparating, so the fiber $F$ is homologous to a surface obtained by collapsing $\gamma$ to a point and this can in turn be thought of as the image of a map $F^{\prime} \stackrel{f}{\rightarrow} E$ where $\chi_{-}\left(F^{\prime}\right)<\chi_{-}(F)$. Hence the image $\pi_{*}[F]$ may be represented by a surface of complexity at most $\chi_{-}\left(F^{\prime}\right)$ (see [7]). We know that any basic class of a circle bundle is a pullback of a class on the base (see [1]). Thus by the adjunction inequality (which still holds for $b_{2}^{+}=1$ ) and equation (1),

$$
\chi_{-}(F)=|K . F|=\left|K . \pi_{*} F\right| \leq \|\left.\pi_{*} F\right|_{T} \leq \chi_{-}\left(F^{\prime}\right)<\chi_{-}(F),
$$

which is a contradiction.

Our proof of Theorem 9 below, which differs from those of [2] and [3], will rely on a theorem of Stallings that characterises fibered 3-manifolds in terms of their fundamental group.

Theorem 8 (Stallings [24]). Let $M$ be a compact, irreducible 3-manifold and suppose there is an extension

$$
1 \rightarrow G \rightarrow \pi_{1}(M) \rightarrow \mathbb{Z} \rightarrow 1
$$

where $G$ is finitely generated and $G \neq \mathbb{Z}_{2}$. Then $M$ fibers over $S^{1}$.

We now come to the main result of this section.

Theorem 9. Let $S^{1} \rightarrow E \stackrel{\pi}{\rightarrow} M$ be a symplectic circle bundle over an irreducible base $M$. If $E$ admits a Lefschetz fibration, then $M$ fibers over $S^{1}$.

Proof. First of all by Lemma 4 we have that $E$ actually admits a fibration $F \rightarrow$ $E \stackrel{p}{\rightarrow} B$. In addition we note that the fiber $\gamma$ of any oriented circle bundle lies in the centre of the fundamental group of the total space. We shall have to consider two distinct cases according to whether $\gamma$ is in the kernel of $p_{*}$ or not.

Case 1. $p_{*}(\gamma) \neq 1$.

Since $\gamma$ was central in the fundamental group of $E$, the fact that $p_{*}(\gamma)$ is nontrivial in $\pi_{1}(B)$ means that $B$ must be a torus. Hence the long exact homotopy sequence of the fibration gives the following short exact sequence:

$$
1 \rightarrow \pi_{1}(F) \rightarrow \pi_{1}(E) \stackrel{p_{*}}{\rightarrow} \pi_{1}\left(T^{2}\right)=\mathbb{Z}^{2} \rightarrow 1 .
$$

Since $M$ is assumed to be irreducible and hence aspherical, we also have the following exact sequence from the homotopy exact sequence of the fibration $S^{1} \rightarrow E \stackrel{\pi}{\rightarrow}$ $M$ :

$$
1 \rightarrow \pi_{1}\left(S^{1}\right)=\langle\gamma\rangle \rightarrow \pi_{1}(E) \stackrel{\pi_{*}}{\rightarrow} \pi_{1}(M) \rightarrow 1 .
$$

Because $\gamma$ is central in $\pi_{1}(E)$, the sequence (2) gives the following exact sequence:

$$
1 \rightarrow \pi_{1}(F) \rightarrow \pi_{1}(E) /\langle\gamma\rangle \stackrel{p_{*}}{\rightarrow} \mathbb{Z}^{2} /\left\langle p_{*}(\gamma)\right\rangle \rightarrow 1 .
$$

Moreover since $p_{*}(\gamma) \neq 1$ we have that $\mathbb{Z}^{2} /\left\langle p_{*}(\gamma)\right\rangle=\mathbb{Z} \oplus \mathbb{Z}_{k}$ for some $k$. If we let $H=p_{*}^{-1}\left(\mathbb{Z}_{k}\right)$ we see that $H$ has $\pi_{1}(F)$ as a finite index subgroup and is thus also 
finitely generated. Then by taking the projection to $\mathbb{Z}$ in the above sequence we obtain

$$
1 \rightarrow H \rightarrow \pi_{1}(E) /\langle\gamma\rangle=\pi_{1}(M) \stackrel{p_{*}}{\rightarrow} \mathbb{Z} \rightarrow 1 .
$$

This is exact and $H \neq \mathbb{Z}_{2}$ since it contains $\pi_{1}(F)$. As $M$ is irreducible, the hypotheses of Theorem 8 are satisfied and we conclude that $M$ fibers over $S^{1}$.

Case 2. $p_{*}(\gamma)=1$.

In this case, $\langle\gamma\rangle \subset \pi_{1}(F)$ and hence $F=T^{2}$. Thus sequence (2) above yields the following:

$$
1 \rightarrow \mathbb{Z}^{2} \rightarrow \pi_{1}(E) \stackrel{p_{*}}{\rightarrow} \pi_{1}(B) \rightarrow 1
$$

and $\langle\gamma\rangle \subset \mathbb{Z}^{2}$. Again by taking the quotient by $\langle\gamma\rangle$ we obtain the following short exact sequence:

$$
1 \rightarrow \mathbb{Z} \oplus \mathbb{Z}_{k}=\mathbb{Z}^{2} /\langle\gamma\rangle \rightarrow \pi_{1}(E) /\langle\gamma\rangle=\pi_{1}(M) \stackrel{p_{*}}{\rightarrow} \pi_{1}(B) \rightarrow 1 .
$$

However since $M$ is irreducible and hence prime and $\pi_{1}(M)$ is infinite, it follows from ([10], Corollary 9.9) that $\pi_{1}(M)$ is torsion free. Hence $k=0$ and $\pi_{1}(M)$ contains an infinite cyclic normal subgroup; thus by (10, Corollary 12.8) it is in fact Seifert fibered and the result follows from Corollary 2 above.

Theorem 9 then allows us to prove Conjecture 1 under the assumption that the total space is a complex manifold.

Corollary 3. Conjecture 1 holds in the case that $E$ is a complex manifold.

Proof. By considering the Kodaira classification and noting that $E$ is spin, symplectic and has $\chi(E)=0$ one concludes that one of the following must hold (cf. [3] Theorem 5.1):

(1) $E=S^{2} \times T^{2}$

(2) $E$ is a $T^{2}$-bundle over $T^{2}$,

(3) $E$ is a Seifert fibration over a hyperbolic orbifold.

If $E=S^{2} \times T^{2}$, then $M=S^{2} \times S^{1}$ and one clearly has an $S^{1}$-invariant symplectic form. In the second case it follows from the argument above that $M$ is a $T^{2}$-bundle over $S^{1}$ and hence has vanishing Thurston norm. In the final case $M$ must be Seifert fibered as in Case 2 in the proof of Theorem 9 and hence the claim holds in the latter two cases by Corollary 2 ,

\section{ACKNowledgments}

The author would like to thank Professor Dieter Kotschick for his wise and patient supervision that culminated in this paper.

\section{REFERENCES}

[1] J. Baldridge, Seiberg-Witten Invariants of 4-manifolds with free circle actions, Communications in Contemporary Math., 3 (2001), 341-353. MR.1849644 (2002d:57024)

[2] W. Chen and R. Matveyev, Symplectic Lefschetz fibrations on $S^{1} \times M^{3}$, Geometry and Topology, 4 (2000), 517-535. MR1800295 (2001j:57034)

[3] T. Etgü, Lefschetz fibrations, complex structures and Seifert fibrations on $S^{1} \times M^{3}$, Algebraic and Geometric Topology, 1 (2001), 469-489. MR1852768 (2002f:57066)

[4] M. Fernández, A. Gray and J. Morgan, Compact symplectic manifolds with free circle actions, and Massey products, Michigan Math. J. 38 (1991), 271-283. MR 1098863 (92e:57049)

[5] S. Friedl and S. Vidussi, Symplectic $S^{1} \times M^{3}$, subgroup separability, and vanishing Thurston norm, J. Amer. Math. Soc. 21 (2008), 597-610. MR2373361 
[6] S. Friedl and S. Vidussi, Symplectic 4-manifolds with a free circle action, preprint arXiv math:GT 08011513 v1 9Jan2008.

[7] D. Gabai, Foliations and the topology of 3-manifolds, J. Diff. Geometry, 18 (1983), 445-503. MR723813 (86a:57009)

[8] R. Gompf and A. Stipsicz, Kirby calculus and the topology of 4-manifolds, Graduate Studies in Mathematics, 20 Amer. Math. Soc., Providence, RI, 1999. MR1707327 (2000h:57038)

[9] A. Hatcher, 3-manifolds, http://www.math.cornell.edu/ hatcher/3M/3Mdownloads.html, 2000.

[10] J. Hempel, 3-manifolds, Princeton University Press, Princeton NJ, $1976 . \quad$ MR0415619 $(54: 3702)$

[11] J. Hempel, Residual finiteness for 3-manifolds, in: Combinatorial Group theory and Topology, (Alta, Utah, 1984), Ann. Math. Stud., 111, (Princeton, NJ, 1987), 379-396. MR895623 (89b:57002)

[12] S. Kojima, Finite covers of 3-manifolds containing essential surfaces of Euler characteristic = 0, Proc. Amer. Math. Soc., 101 (1987), 743-747. MR911044(89b:57010)

[13] D. Kotschick, Orientations and geometrisations of compact complex surfaces, Bull. London Math. Soc. 29 (1997), 145-149. MR.1425990 (97k:32047)

[14] D. Kotschick, Free circle actions with contractible orbits on symplectic manifolds, Math. Zeitschrift, 252 (2006), 19-25. MR2209149 (2006j:57055)

[15] P. Kronheimer, Embedded surfaces and gauge theory in three and four dimensions, in Surveys in Differential geometry, V. III, (Cambridge, MA 1996), 243-298, Int Press, 1998. MR1677890 (2000a:57086)

[16] P. Kronheimer and T. Mrowka, Monopoles and three-manifolds, Cambridge Univ. Press, 2007. MR2388043

[17] C. Lescop, Global surgery formula for the Casson-Walker invariant, Princeton University Press, Princeton, NJ, 1996. MR1372947 (97c:57017)

[18] T.J. Li and A. Liu, Symplectic structure on ruled surfaces and a generalized adjunction formula, Math. Res. Letters 2 (1995), 453-471. MR1355707 (96m:57052)

[19] A. Liu, Some new applications of general wall crossing formula, Gompf's conjecture and its applications, Math. Res. Letters 3 (1996), 569-585. MR.1418572 (97k:57038)

[20] J. D. McCarthy, On the asphericity of a symplectic $M^{3} \times S^{1}$, Proc. Amer. Math. Soc., Vol. 129, no. 1 (2001), 257-264. MR 1707526 (2001c:57024)

[21] J. Morgan and G. Tian, Ricci flow and the Poincaré conjecture, Clay Mathematics Monographs 3, Providence RI, 2007. MR2334563 (2008d:57020)

[22] G. Perelman, The entropy formula for the Ricci flow and its geometric applications, Preprint arXiv: math.DG/0211159 v1 11Nov2002.

[23] G. Perelman, Ricci flow with surgery on three-manifolds, Preprint arXiv: math.DG/0303109 v1 10Mar2003.

[24] J. Stallings, On fibering certain 3-manifolds, Topology of 3-manifolds and related topics, Prentice Hall, NJ (1962), 95-100. MR0158375 (28:1600)

[25] C.H. Taubes, The Seiberg-Witten invariants and symplectic forms, Math. Res. Letters 1 (1994), 809-822. MR1306023 (95j:57039)

[26] C.H. Taubes, More constraints on symplectic forms from Seiberg-Witten invariants, Math. Res. Letters 2 (1995), 9-13. MR.1312973 (96a:57075)

[27] W.P. Thurston, A norm for the homology of 3-manifolds, Mem. Amer. Math. Soc. 59 (1986), no. 339, i-vi and 99-130. MR823443 (88h:57014)

[28] V. Turaev, Introduction to combinatorial torsions, Birkhäuser, Basel, 2001. MR1809561 (2001m:57042)

Mathematisches Institut, Ludwig-Maximilians-Universität, Theresienstr. 39,80333 MünChen, Germany

E-mail address: jonathan.bowden@mathematik.uni-muenchen.de 